Reprod. Nutr. Dévelop., 1981, 21 (1), 83-93.

\title{
Etude de l'utilisation digestive d'une cellulose purifiée chez la truite arc-en-ciel (Salmo gairdneri) et la carpe commune (Cyprinus carpio)
}

\author{
par Françoise BERGOT \\ avec la collaboration technique de Jeannine BREQUE
}

Laboratoire de Nutrition des poissons, I.N.R.A.

Saint-Pée-sur-Nivelle 64310 Ascain, France.

Summary. Digestibility of a purified cellulose by the rainbow trout (Salmo gairdneri) and the common carp (Cyprinus carpio).

A sem i-purified diet containing 22 p. 100 of a wood cellulose extract without lignin but still containing 22 p. 100 of hemicelluloses was distributed for one month to rainbow trout and common carp reared at 17 and $20^{\circ} \mathrm{C}$, respectively. The digestibility of the main dietary constituents was determined by an indirect method using chrome oxide as an inert tracer. The feces were recovered by a continuous automatic collector which rapidly removed them from the water, minimizing alteration by leaching. The cellulose content was estimated by the Weende (crude fiber) and the Van Soest (neutral detergent fiber and acid detergent fiber) methods.

The digestibility coefficients obtained for trout as well as for carp indicate that cellulose and hemicelluloses were not digested. In both species, volatile fatty acid concentration in the different segments of the digestive tract was low (less than $10 \mathrm{mM} / \mathrm{l}$ ). These results lead us to suggest that trout and carp cannot degrade purified cellulose.

\section{Introduction.}

La cellulose, les hémicelluloses et la lignine sont présentes dans les végétaux aquatiques qu'ingèrent les poissons herbivores et dans les céréales, les tourteaux et les issues de meunerie des aliments destinés aux poissons d'élevage tels que les truites et les carpes. Peu de données sont disponibles concernant leur utilisation digestive et des divergences existent suivant les auteurs. Ainsi, Austreng (1977) chez la Truite ef Buddington (1979) chez le Tilapia pensent que la cellulose est totalement indigestible. A l'opposé Smith (1971) signale que la digestibilité de l'a-cellulose chez la Truite est de 13 p. 100.

Les vertébrés ne possèdent pas de cellulase endogène ef l'on admet que la dégradation des matières cellulosiques est le fait des fermentations dues aux microorganismes présents dans le tube digestif. Les poissons, tant marins que d'eau douce, possèdent une microflore intestinale. Elle est quantitativement moins abondante que celle des vertébrés homéothermes : $10^{2}$ à $10^{8}$ bactéries/g dans le tube digestif (Trust et Sparrow, 1974) contre plus de $10^{10}$ dans le cæcum des mammifères (Ducluzeau, 1969). 
Elle est qualitativement différente, la plupart des bactéries étant aérobies ou anaérobies facultatives (Trust et Sparrow, 1974 ; Horsley, 1977). Une activité cellulolytique a été signalée dans les contenus digestifs de différents poissons. Selon les auteurs, cette activité serait soił due à la microflore intestinale (Stickney et Shumway, 1974) soit issue des proies consommées par les poissons (Prejs et Blaszczyk, 1977 ; Niederholzer et Hofer, 1979).

Ces résultats nous ont montré qu'il était nécessaire de mieux connaître l'imporfance de la digestion de la cellulose chez le poisson. Nous avons réalisé ce travail chez deux espèces d'intérêt économique : la truite arc-en-ciel (Salmo gairdneri) espèce carnivore et la carpe commune (Cyprinus carpio), espèce omnivore à tube digestif plus long que celui de la truite. Nous avons mesuré à partir d'un même aliment contenant 22 p. 100 d'une cellulose purifiée (sans lignine) la digestibilité de cette cellulose et celle des autres principaux constituants du régime. En outre, nous avons étudié les produits terminaux de la fermentation microbienne dans les contenus digestifs prélevés au moment de l'abattage des animaux.

\section{Matériel et méthodes.}

1) Aliment (tabl. 1). - L'aliment utilisé contient 22 p. 100 d'une cellulose en poudre extraite du bois et délignifiée. L'hydrolyse par l'acide sulfurique à 72 p. 100 ne laisse pas de résidu ce qui traduit l'absence totale de lignine. Cette cellulose contient des hémicelluloses essentiellement sous forme de xylose (20 p. 100, tabl. 2), mais très peu de constituants non glucidiques (traces d'azote et de sels minéraux ; 0,2 p. 100 de résine ; 0,35 p. 100 de cendres).

TABLEAU 1

Composition de l'aliment (p. $100 \mathrm{MS}$ )

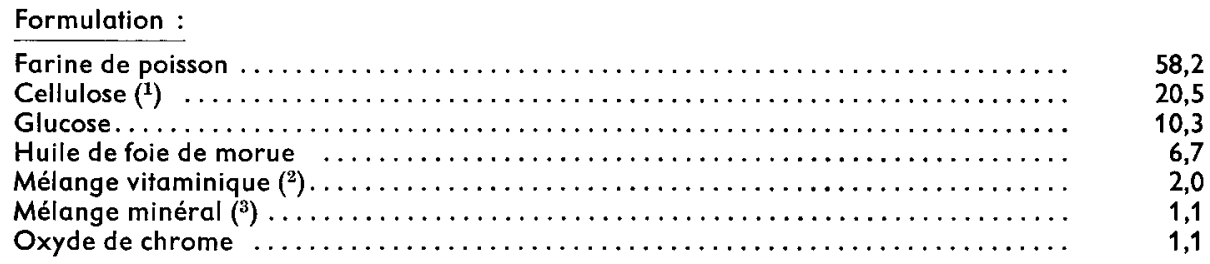

Composition chimique :

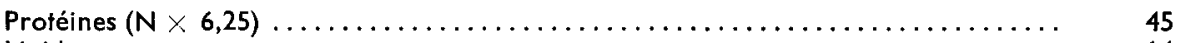

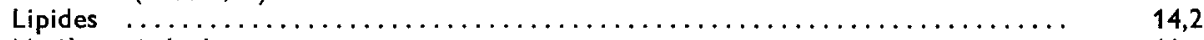

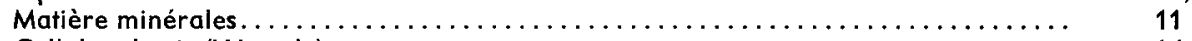

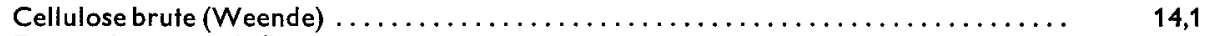

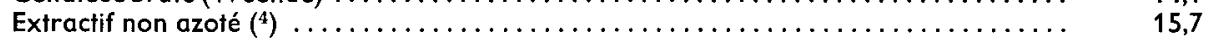

(1) Cellulose Durieux (CEPO SS/200); $\left({ }^{2}\right)$ EIFAC (1971). Ce mélange, fait sur un support de cellulose incorporée au taux de 68 p. 100, apporte 1,4 p. 100 de cellulose. Le pourcentage théorique de cellulose dans l'aliment s'élève donc à 21,9 p. $100 ;\left({ }^{3}\right)$ Luquet (1971); $\left(^{4}\right)$ ENA = Mat. organique (mat. azotées + mat. grasses + cellulose brute). 
TABLEAU 2

Caractéristiques de la cellulose utilisée

Déterminations effectuées

Résultats

Méthodes d'analyse utilisées

Dimensions des fibres $(\mu)$ :

- longueur $\max \ldots \ldots \ldots \ldots \ldots \ldots \ldots$

- Iongueur moyenne .............

- diamètre $\max \ldots \ldots \ldots \ldots \ldots \ldots \ldots \ldots$

- diamètre moyen

150

50

Composition chimique (p. $100 \mathrm{MS}$ ) :

- « cellulose $»$ totale. . . . . . . . . . . .

- $\alpha$-cellulose $\ldots \ldots \ldots \ldots \ldots \ldots \ldots \ldots$

- $\beta$-cellulose $\ldots \ldots \ldots \ldots \ldots \ldots \ldots$

- $\gamma$-cellulose

- fraction NDF $\left(^{1}\right)$

- fraction ADF $\left.{ }^{2}\right) \ldots \ldots \ldots \ldots \ldots$

- lignine sulfurique $\ldots \ldots \ldots \ldots \ldots \ldots$

- cellulose brute ...............

- arabinose .................

- xylose ....................

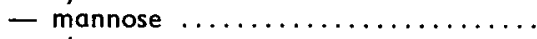

- glucose ...................
45

20

Examen microscopique.

\begin{tabular}{|c|c|}
\hline $\begin{array}{l}99,5 \\
80\end{array}$ & $\begin{array}{l}\text { Fraction insoluble dans } \mathrm{NaOH} 17,5 \text { p. } \\
100\end{array}$ \\
\hline 9 & $\begin{array}{l}\text { Fraction soluble dans } \mathrm{NaOH} 17,5 \mathrm{p} \text {. } \\
100 \text { et reprécipitée par } \mathrm{CH}_{3} \mathrm{COOH} \text {. }\end{array}$ \\
\hline 11 & $\begin{array}{l}\text { Fraction soluble dans } \mathrm{NaOH} 17,5 \mathrm{p} \text {. } \\
100 \text { ef non reprécipitable. }\end{array}$ \\
\hline $\begin{array}{c}97,6 \\
77,1 \\
0 \\
64\end{array}$ & $\begin{array}{l}\text { Van Soest et Wine (1967). } \\
\text { Van Soest (1963). } \\
\text { Van Soest (1963). } \\
\text { Weende. }\end{array}$ \\
\hline $\begin{array}{r}0,7 \\
20,4 \\
1,4 \\
71,1\end{array}$ & $\begin{array}{l}\text { Chromatographie en phase gazeuse } \\
\text { des sucres libérés par hydrolyse acide } \\
\text { ef injectés sous forme de dérivés aldi- } \\
\text { tols acétylés (étalon interne inositol) } \\
\text { (Sawardeker et al., 1965). }\end{array}$ \\
\hline
\end{tabular}

(1) Neutral Detergent Fiber ; $\left({ }^{2}\right)$ Acid Detergent Fiber.

Les aliments ont été fabriqués au laboratoire afin de connaître avec précision les quantités de cellulose incorporée. En effet, le dosage des glucides des parois végétales par les méthodes de Van Soest, possible dans les fèces, est très difficile à effectuer dans l'aliment en raison de sa forte teneur en protéines et en lipides.

2) Animaux et mode d'alimentation. - L'expérience sur carpes a été faite en été (juillet, août), celle sur truites en automne (octobre, novembre). Ces poissons provenaient d'une pisciculture expérimentale (Donzacq, Landes) alimentée par une eau de source à température constante $\left(17^{\circ} \mathrm{C}\right)$. Ils ont été répartis dans 4 bacs à digestibilité à raison de 10 poissons par bac (poids moyen initial de $130 \mathrm{~g}$ pour les truites et $270 \mathrm{~g}$ pour les carpes). Ils ont été adaptés à ce dispositif pendant 15 jours, durée pendant laquelle ils ont reçu un aliment de type commercial. Puis ils ont reçu quotidiennement l'aliment expérimental sous forme de granulés secs pendant 30 jours (truites) ou 31 jours (carpes). Cet aliment a été distribué au rythme de 2 repas par jour ( $9 \mathrm{~h}-16 \mathrm{~h} \mathrm{30}$ ). Les carpes n'ont cependant reçu qu'un seul repas les samedis et dimanches. Les granulés ont été distribués lentement afin qu'ils puissent être immédiatement consommés par les poissons et ne subissent pas de modification au contact de l'eau. Les quantités distribuées ont été enregistrées chaque jour. Les plateaux de récolte des fèces ont été changés chaque matin avant le repas de $9 \mathrm{~h}$. Les fèces recueillies ont été conservées à 
- $20^{\circ} \mathrm{C}$ puis lyophilisées. La température de l'eau a été enregistrée quotidiennement (fig. 1). Elle a été en moyenne de $17^{\circ} \mathrm{C}$ pour les truites et de $20^{\circ} \mathrm{C}$ pour les carpes.

3) Dispositif de récolte des fèces. - Avec les truites nous avons utilisé d'une part le collecteur automatique de fèces mis au point au laboratoire (Choubert et al., 1979) ef d'autre part un système légèrement modifié où les passoires sont remplacées par des palettes métalliques de maille plus fine $(650 \mu)$, animées d'un mouvement de translation linéaire qui soustraił encore plus rapidement les fèces à l'action de l'eau (Choubert, en préparation). Les fèces de carpes ont toutes été récoltées par le premier procédé.

4) Prélèvement des contenus digestifs. - Dans le cas des truites, les poissons ont été sacrifiés dès la fin de l'expérience de digestibilité. Dans le cas des carpes, les poissons ont été maintenus dans leurs bacs et ont continué à recevoir l'aliment expérimental durant une période pendant laquelle la température de l'eau a atteint $25^{\circ} \mathrm{C}$. Le sacrifice des animaux (carpes et truites) a été effectué entre 3 et 8 h après l'administration d'un repas. Les contenus digestifs ont été prélevés à 4 niveaux pour les truites (estomac, intestin antérieur, moyen, postérieur) et à 3 niveaux seulement pour les carpes qui sont dépourvues d'estomac (intestin antérieur, moyen, postérieur). Les échantillons ont été stockés à $-20^{\circ} \mathrm{C}$.

5) Analyses. - La matière sèche des aliments et des fèces a été déterminée à l'étuve à $105^{\circ} \mathrm{C}$ jusqu'à poids constant, les matières minérales par calcination dans un four à moufle $\left(550^{\circ} \mathrm{C}\right.$ ) pendant une nuit, l'oxyde de chrome par la méthode de Bolin et al. (1952), les lipides totaux par la méthode de Folch et al. (1957), les protéines par la méthode de $\mathrm{Kjeldahl}(\mathrm{N} \times 6,25)$. La cellulose brute a été dosée selon la méthode de Weende avec un appareil de dosage automatique Fibertec (Tecator LTD) sur creusets frittés de porosité 2.

La défermination des fractions NDF (Neutral Detergent Fiber) et ADF (Acid Detergent Fiber), selon Van Soest et Wine (1967) et Van Soest (1963) a été faite sur les fèces et sur la cellulose native mais pas sur l'aliment. Compte tenu de l'absence de lignine et de substances pectiques dans nos échantillons, nous avons considéré que les fractions NDF et ADF, débarrassées des protéines et des minéraux, pouvaient être assimilées respectivement à l'ensemble hémicelluloses + cellulose et à la cellulose vraie.

L'observation au microscope de la cellulose ayant révélé la présence de très fines particules ( 5 à $10 \mu$ de diamètre pour certaines fibres), nous avons utilisé des creusets frittés de fine porosité ( $\left.n^{\circ} 4\right)$ protégés par un filtre Whatman en fibre de verre GFA retenant les particules supérieures à $1,6 \mu$. Nous avons vérifié que ces filtres étaient bien constifués uniquement de substances minérales. Tout échantillon ayant nécessité un temps de filtration supérieur à $10 \mathrm{~min}$ a été éliminé car une filtration lente due à un colmatage partiel du filtre par des substances organiques (protéiques par exemple) conduit presque toujours à un résultat par excès (Giger et al., 1979).

Les extractions neutre et acide ont été pratiquées de façon séquentielle. L'attaque par le détergent acide a ainsi porté sur le résidu NDF et non sur l'échantillon de départ.

Afin de suivre l'évolution de la digestibilité de l'aliment au cours du temps, nous avons dosé l'oxyde de chrome sur les échantillons de fèces recueillies quotidienne- 
ment. Pour les truites, ces dosages ont été faits du $11 \mathrm{e}$ au $30 \mathrm{e}$ jour d'expérience (sur les 4 bacs) et pour les carpes du 1 er jour au 31e jour (sur 2 des 4 bacs). Malgré des fluctuations importantes de consommation suivant le jour et le bac considérés (coefficient de variation $=$ écart-type/moyenne $=34 \mathrm{p} .100$ pour les carpes et les iruites) le CUD de la matière sèche varie peu ( $C V=3$ p. 100 chez les deux espèces) (fig. 1). D'autre part nous n'avons pas trouvé de corrélation significative entre la consommation d'un bac un jour donné ef le CUD de la matière sèche le même jour ni le jour suivant. Afin de disposer d'une quantité d'échantillon suffisante pour l'ensemble des dosages. nous avons regroupé pour chaque bac (4 chez chaque espèce) les fèces de plusieurs jours. Pour les truites, les dosages ont porté sur les fèces émises de J12 à J17 ef de J24 à $\mathrm{J} 30$ et pour les carpes sur celles émises de J21 à $\mathrm{J} 31$.
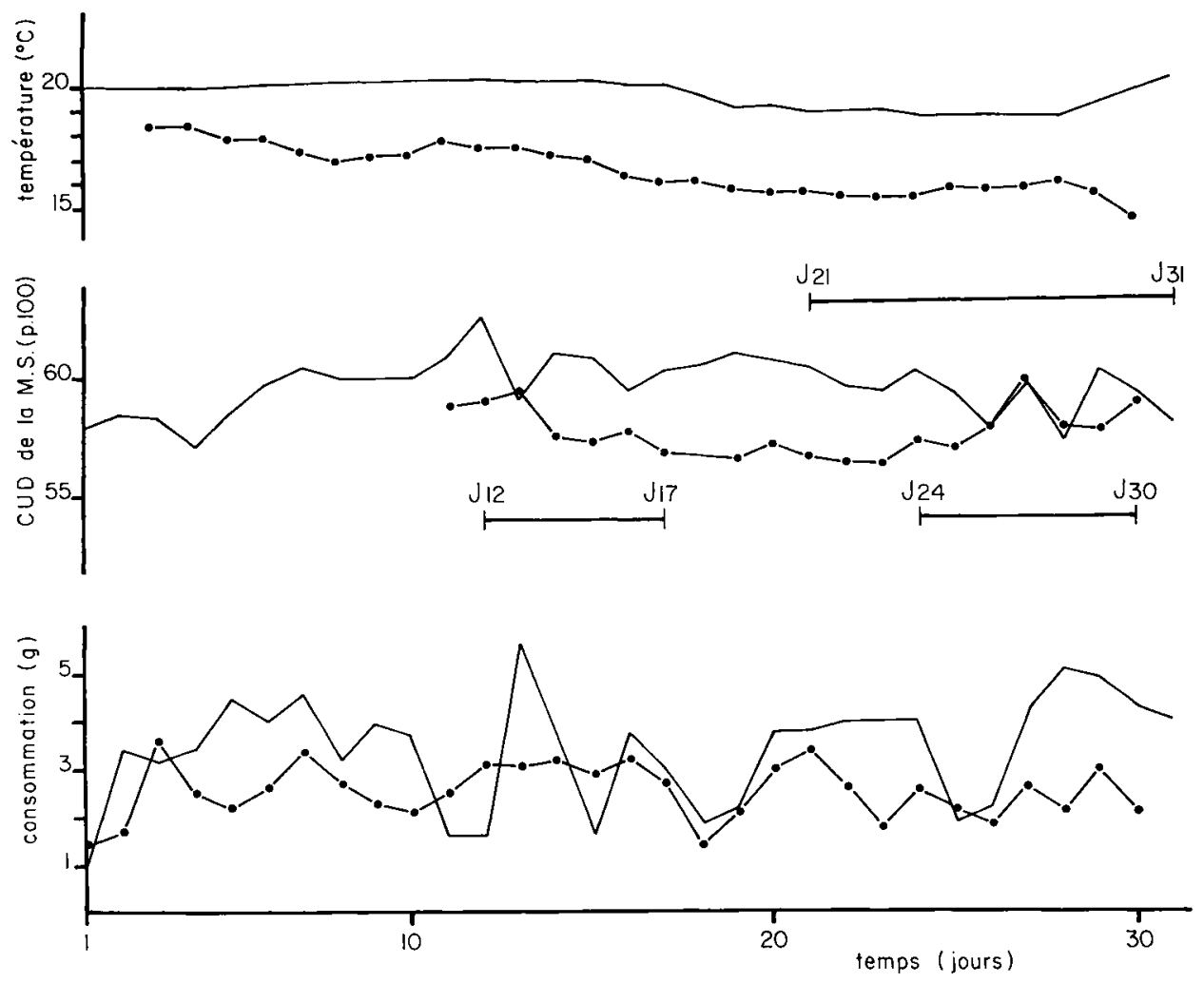

FIG. 1. - Variations de la température, de la consommation et de la digestibilité de la matière sèche ou cours de l'expérience (la consommation journalière est exprimée en grammes par jour par poisson). Carpe (—), Truite $(-\bullet)$.

Après décongélation, les échantillons de contenus digestifs prélevés au moment de l'abattage ont été centrifugés. Le $\mathrm{pH}$ a été mesuré dans les surnageants qui ont ensuite été traités par un mélange conservateur $\left(\mathrm{HgCl}_{2}, 1\right.$ p. $100 ; \mathrm{H}_{3} \mathrm{PO}_{4}, 5$ p. 100) 
pour empêcher le développement des fermentations. Les acides gras volatils (AGV) ont été dosés par chromatographie en phase gazeuse selon la méthode de Jouany (1978).

\section{Résultats.}

1) Composition des fèces (tabl. 3). - La composition des fèces de truite est peu différente de celle de carpe. Leur teneur en matières minérales est élevée ( 20 à 24 p. 100). La matière organique est relativement pauvre en protẻines (15 à 16 p. 100) et surtout en lipides (2 à 4 p. 100). Elle est essentiellement composée de glucides.

\section{TABLEAU 3}

Composition des fèces de truifes ef de carpes en p. $100 \mathrm{MS}$ (moyenne \pm écart type de la moyenne).

\begin{tabular}{|c|c|c|}
\hline $\begin{array}{l}\text { Espèce } \\
\text { Nombre de lots }\end{array}$ & $\begin{array}{l}\text { Truite } \\
\mathbf{n}=\mathbf{8}\end{array}$ & $\begin{array}{l}\text { Carpe } \\
n=4\end{array}$ \\
\hline 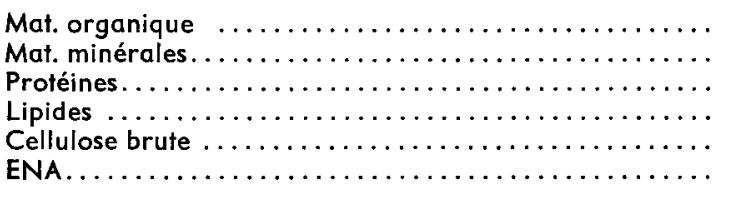 & $\begin{array}{r}76 \pm 0,2 \\
24 \pm 0,2 \\
16 \pm 0,2 \\
4 \pm 0,2 \\
35 \pm 0,6 \\
21 \pm 0,7\end{array}$ & $\begin{array}{r}80 \pm 0,6 \\
20 \pm 0,6 \\
15 \pm 0,4 \\
2 \pm 0,1 \\
40 \pm 0,3 \\
23 \pm 0,6\end{array}$ \\
\hline 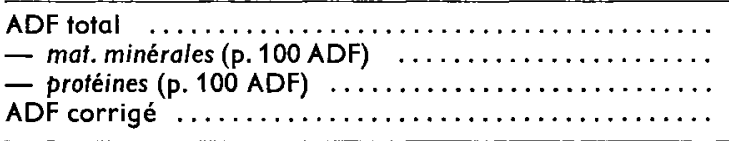 & $\begin{array}{l}46 \pm 0,5 \\
10 \pm 0,2 \\
0,8 \pm 0,1 \\
41 \pm 0,5\end{array}$ & $\begin{array}{l}51 \pm 0,6 \\
5 \pm 0,3 \\
0,4 \pm 0,1 \\
48 \pm 0,4\end{array}$ \\
\hline 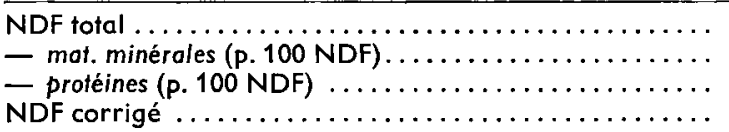 & $\begin{array}{r}57 \pm 0,6 \\
10 \pm 0,9 \\
2 \pm 0,1 \\
51 \pm 0,6\end{array}$ & $\begin{aligned} 65 & \pm 0,5 \\
5 & \pm 0,6 \\
2 & \pm 0,1 \\
60 & \pm 0,4\end{aligned}$ \\
\hline
\end{tabular}

La fraction NDF totale représente le principal constituant des fèces de truite, soit près de 60 p. 100 de la matière sèche. Cette fraction contient 10 p. 100 de matières minérales et 2 p. 100 de protéines. Les 88 p. 100 restant constituent la partie glucidique du NDF (cellulose + hémicelluloses ou NDF corrigé) qui représente ainsi environ 50 p. 100 de la matière sèche des fèces. La fraction ADF totale représente 46 p. 100 des fèces. Elle contient 10 p. 100 de matières minérales mais est pratiquement dépourvue de protéines ( $<1$ p. 100). La partie glucidique de l'ADF (cellulose vraie ou ADF corrigé) représente ainsi 41 p. 100 de la matière sèche des fèces.

Les teneurs en NDF total et ADF total des fèces sont légèrement plus élevées chez la carpe que chez la truite et représentent respectivement 65 et 51 p. 100 de la matière sèche. Ces fractions contiennent des protéines en proportion comparable à celle des truites mais moins de matières minérales ( 5 p. 100 au lieu de 10 p. 100). Les fractions NDF et ADF corrigées représentent ainsi respectivement 60 et 48 p. 100 de la matière sèche des fèces. 
La cellulose brute des fèces, légèrement plus élevée chez la carpe (40 p. 100) que chez la truite (35 p. 100), représente chez ces deux espèces la même proportion de la fraction ADF totale (76 à 78 p. 100).

2) Digestibilité (tabl. 4). - Chez les deux espèces, le CUD de la matière organique est de l'ordre de 65 p. 100. La digestibilité des protéines et des lipides est élevée. Elle est supérieure chez la carpe où les CUD atteignent 88 p. 100 pour les protéines et 95 p. 100 pour les lipides contre 85 et 87 p. 100 respectivement chez la truite. La digestibilité des matières minérales est faible et de l'ordre de 30 p. 100 dans les deux cas.

TABLEAU 4

Coefficients d'utilisation digestive apparents (CUDa) des différents constifuants de l'aliment chez les truites ef les corpes (moyenne \pm écart-type de la moyenne)

\begin{tabular}{|c|c|c|c|}
\hline \multirow{2}{*}{$\frac{\text { Espèce }}{\text { Nombre de lots }}$} & \multicolumn{2}{|c|}{ Truite } & \multirow{2}{*}{$\frac{\text { Carpe }}{n=4}$} \\
\hline & $\begin{array}{c}n=4 \\
\text { 1re période * }\end{array}$ & $\begin{array}{c}n=4 \\
\text { 2e période * }\end{array}$ & \\
\hline 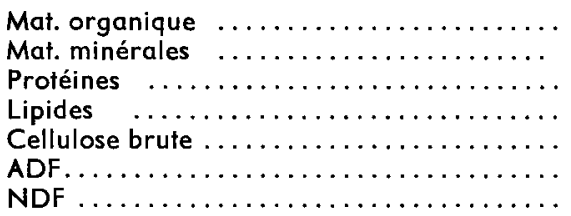 & $\begin{array}{r}64 \pm 0,5 \\
30 \pm 0,6 \\
85 \pm 0,4 \\
87 \pm 1,2 \\
-4 \pm 3,8 \\
-2 \pm 1,4 \\
1 \pm 1,6\end{array}$ & $\begin{array}{r}64 \pm 0,5 \\
28 \pm 1,3 \\
85 \pm 0,4 \\
86 \pm 1,5 \\
1 \pm 2,6 \\
-6 \pm 1 \\
-4 \pm 0,8\end{array}$ & $\begin{array}{r}67 \pm 0,4 \\
31 \pm 1,8 \\
88 \pm 0,3 \\
95 \pm 0,2 \\
-5 \pm 0,6 \\
-5 \pm 1,4 \\
-4 \pm 1,1\end{array}$ \\
\hline
\end{tabular}

* 1re période $\left(J_{12^{-}} J_{17}\right), 2^{2}$ période $\left(J_{24}-J_{30}\right)$.

Chez les deux espèces, les résultats indiquent que la cellulose étudiée n'est pas digestible. Chez la truite, pour les deux périodes considérées et pour les deux systèmes de récolte employés, l'estimation moyenne du CUD de la cellulose brute ne diffère pas significativement de zéro. II en est de même pour le CUD du NDF corrigé. Le CUD moyen de l'ADF corrigé est légèrement négatif. Chez la carpe les valeurs obtenues pour les CUD des différentes fractions (cellulose bruie, NDF et ADF corrigés) sont en moyenne négatives et proches de zéro $(-5 \mathrm{p}$. 100). Ces résultats expliquent la faible digestibilité de la matière organique.

3) Acides gras volatils des contenus digestifs (tabl. 5). - La concentration totale en AGV des contenus digestifs est faible chez les deux espèces ef inférieure à $10 \mathrm{mM} / 1$. Parmi les AGV, c'est l'acide acétique $\left(C_{2}\right)$ qui est le plus abondant. II représente 60 p. 100 des AGV totaux chez la truite et près de 80 p. 100 chez la carpe. Les acides propionique $\left(C_{3}\right)$ et butyrique $\left(C_{4}\right)$ représentent chacun en moyenne 10 p. 100 des AGV chez la carpe et 15 p. 100 chez la truite. Les acides valérique $\left(C_{5}\right)$ et caproïque $\left(C_{6}\right)$ sont présents en très faible proportion $(<5$ p. 100). Il faut signaler la présence d'acide isovalérique ( 3 à 4 p. 100) tandis que l'acide isobutyrique est pratiquement absent. Les valeurs du $\mathrm{pH}$ sont de l'ordre de 4 dans l'estomac de la truite et comprises entre 7,5 et 8,5 dans les différents segments de l'intestin de carpe et de truite. 
TABLEAU 5

$\mathrm{pH}$ ef concentration en AGV des confenus digestifs de truites et de carpes

\begin{tabular}{|c|c|c|c|c|c|c|c|c|c|}
\hline & \multirow{2}{*}{$\mathrm{pH}$} & \multirow{2}{*}{$\begin{array}{c}\text { Acidité totale } \\
\mathrm{mM} / \mathrm{I}\end{array}$} & \multicolumn{7}{|c|}{ Pourcentage molaire des AGV } \\
\hline & & & $\mathrm{C}_{2}$ & $\mathrm{C}_{3}$ & $\mathrm{C}_{\mathrm{i}}$ & $\mathrm{iC}_{4}$ & $\mathrm{C}_{5}$ & $\mathrm{iC}_{5}$ & $c_{\theta}$ \\
\hline \multicolumn{10}{|l|}{ Truife $(n=3)$} \\
\hline Estomac.... & 4,0 & $7,6 \pm 0,7$ & 63 & 17 & 12 & $<1$ & 2 & 3 & 2 \\
\hline $\mathrm{IA}^{*} \ldots \ldots \ldots \ldots$ & 8,0 & $3,6 \pm 0,8$ & 62 & 16 & 11 & 0 & 4 & 4 & 4 \\
\hline $\mathbb{I} \mid \ldots \ldots \ldots \ldots \ldots$ & 8,0 & $3,0 \pm 0,4$ & 62 & 15 & 12 & 0 & 5 & 4 & 4 \\
\hline IP $\ldots \ldots \ldots \ldots \ldots$ & 8,5 & $4,3 \pm 1,8$ & 56 & 18 & 13 & $<1$ & 6 & 4 & 4 \\
\hline \multicolumn{10}{|l|}{ Carpe $(n=4)$} \\
\hline IA $\ldots \ldots$ & 7,7 & $3,3 \pm 0,4$ & 78 & 9 & 7 & $<1$ & 1 & 4 & 1 \\
\hline $\mathbb{M} \ldots \ldots$ & 7,5 & $7,5 \pm 1,2$ & 77 & 11 & 8 & $<1$ & 1 & 3 & 1 \\
\hline IP $\ldots$ & 8,0 & $8,3 \pm 1,7$ & 76 & 10 & 8 & $<1$ & 1 & 3 & 2 \\
\hline
\end{tabular}

* IA, IM, IP, intestin antérieur, moyen postérieur.

\section{Discussion.}

La cellulose que nous avons utilisée est une cellulose cristalline purifiée, c'est-àdire délignifiée. D'après Van Soest (1973) et Fahey (1979) les celluloses extraites, bien que totalement dégradables car exemptes de lignine sont attaquées plus lentement que les substrats naturels. En outre, l'attaque d'une cellulose cristalline, donc totalement insoluble, nécessite la présence de cellulases vraies, (« $C_{1}$-cellulases 》) moins répandues que les poly- $\beta-1-4$-glucosidases (Halliwell, 1959 ; Lang et Briggs, 1976), qui ne peuvent attaquer que les celluloses solubles ( $C M$-cellulose par exemple). Ces considérations pourraient expliquer en partie les résultats que nous avons obtenus concernant l'incapacité de la truite et de la carpe à dégrader la cellulose. Cependant, Henry et Etienne (1969) utilisant comme nous une cellulose extraite du bois (cellulose Colmacel) ont observé chez le porc une digestibilité variant de 30 à 45 p. 100 suivant l'âge des animaux. De même chez le mouton, la même cellulose incorporée à raison de 30 p. 100 dans un régime semi-synthétique est digérée à raison de 60 à 70 p. 100 selon l'âge de l'animal et peut être presque totalement dégradée lorsqu'elle est in cubée in vitro pendant 16 heures en présence de jus de rumen ( $M$. Durand, communication personnelle).

Nos résultats sont en accord avec ceux d'Austreng (1977) qui, bien qu'utilisant une source de cellulose très différente de la nôtre (farine d'herbe et d'algues marines) considère que la cellulose brute n'est pas digestible et qu'elle peut être utilisée comme marqueur dans les expériences de digestibilité chez la truite. Les valeurs légèrement négatives que nous avons observées pour les CUD des fractions cellulose brute, NDF et ADF corrigés, peuvent s'expliquer par des erreurs expérimentales au niveau de la fabrication de l'aliment et au niveau des dosages. Tandler et Beamish (1979) indiquent également une digestibilité négative de l'a-cellulose chez Micropterus salmoides $(-8 \pm 4$ p. 100). Du fait des valeurs légèrement négatives que nous avons obtenues 
nous n'avons pas attribué de signification à l'estimation de la digestibilité de la fraction hémicelluloses (différence NDF-ADF).

Les valeurs de $\mathrm{pH}$ et de concentration en acides gras volatils que nous avons observées dans les contenus digestifs sont en accord avec les valeurs indiquées par Paris ef al. (1977) chez les mêmes espèces. Les teneurs en AGV ( $<10 \mathrm{mM} / \mathrm{l})$, très faibles par rapport à celles que l'on observe chez les mammifères même non ruminants, peuvent indiquer la présence d'une microflore mais n'impliquent pas nécessairement la dégradation de la cellulose. Les $A G V$ peuvent en effet provenir d'autres constituants du régime. Chez le veau préruminant ne recevant que du lait, Assan (1974) observe des teneurs en AGV de l'ordre de $30 \mathrm{mM} / \mathrm{l}$ dans l'iléon et des valeurs dépassant $150 \mathrm{mM} / \mathrm{l}$ dans le côlon.

La flore des poissons est plus variable que celle des mammifères et est influencée quantitativement et qualitativement par la température et par la microflore de l'eau (Lesel, 1979). On peut donc envisager la présence, dans certains cas, d'une flore cellulolytique dans le tube digestif. Ceci expliquerait que Smith (1971) ait obtenu un CUD de 13 p. 100 pour l' $\alpha$-cellulose chez la truite et que Buhler et Benville (cités par Smith, 1971) aient recueilli du $\mathrm{CO}_{2}$ marqué chez des truites ayant ingéré de la cellulose radioactive, ce phénomène n'étant plus observé après l'administration d'antibiotiques.

Nos résultats indiquent une activité cellulolytique de la flore digestive extrêmement réduite ou nulle chez la truite et la carpe. Niederholzer et Hofer (1979) estiment que, chez le gardon ef le rotengle, espèces relativement herbivores, l'activité cellulolytique existante permet tout au plus de rompre les membranes des cellules végétales mais est insuffisante pour hydrolyser la cellulose. Trust ef al. (1979) ne décelant pas d'activité cellulolytique chez la carpe chinoise qui consomme de grandes quantités de végétaux et est utilisée à ce titre dans le faucardage des étangs, concluent que cette espèce se contente de broyer les végétaux pour en extraire les substances solubles (protéines, sucres) et laisse intacts la cellulose et les autres polyosides.

En conclusion, il apparaît que les poissons sont de très mauvais utilisateurs des glucides des parois végétales. Trust et al. (1979) suggèrent que l'implantation d'une flore cellulolytique efficace nécessite à la fois une température élevée et un compartiment où peuvent stagner les contenus digestifs afin que la vitesse de multiplication des bactéries compense leur évacuation par le péristaltisme. Chez les vertébrés poecilothermes ce point de vue est confirmé par le fait que la tortue verte marine Chelonia mydas, qui possède une zone dilatée entre l'intestin grêle et le gros intestin, utilise à $30^{\circ} \mathrm{C}$ la cellulose aussi bien qu'un ruminant (Bjorndal, 1979 ; Fenchel et al., 1979). En ce qui concerne les poissons étudiés jusqu'ici, les circonstances favorables à la cellulolyse ne semblent pas réunies.

Reçu en mai 1980.

Accepté en septembre 1980.

Remerciements. - Je remercie $P$. Thivend pour ses critiques et ses suggestions lors de la rédaction de ce travail. Je remercie également J. P. Jouany pour le dosage des acides gras volatils, J. M. Brillouet pour l'analyse par chromatographie en phase gazeuse des pentoses ef des hexoses constitutifs de la cellulose Durieux et B. Elhorga pour les soins apportés aux animaux. 


\section{Références}

ASSAN B. E., 1974. Contribution à l'étude de la digestion intestinale chez le veau préruminant. Th. Doct.Ing. Univ. Clermont-Ferrand. 81 pp.

AUSTRENG E., 1977. Faf and protein in diets for salmonoid fishes. IV. Protein content in dry diets for salmon parr (Salmo salar, L.). Meld. Nor. Landbrukshoqsk., 56, 10 pp. (en norvégien).

BJORNDAL K. A., 1979. Cellulose digestion and volatile fatty acid production in the green turtle, Chelonia mydas. Comp. Biochem. Physiol., 63A, 127-133.

BOLIN D. W., KING R. P., KLOSTERMAN W. W., 1952. A simplified method for the determination of chromic oxide $\left(\mathrm{Cr}_{2} \mathrm{O}_{3}\right)$ when used as an inert substance. Science, 116, 634-635.

BUDDINGTON R. K., 1979. Digestion of an aquatic macrophyte by Tilapia zillii Gervais. J. Fish Biol., 15, 449-455.

CHOUBERT G. Jr, DE LA NOUË J., LUQUET P., 1979. Continuous quantitative automatic collector for fish feces. Progve Fish Culf., 41, 64-67.

DUCLUZEAU R., 1969. Influence de l'espèce zoologique sur la microflore du tractus gastrointestinal. Rev. Immunol., 33, 345-384.

EIFAC, 1971. Salmon and trout feeds and feeding. EIFAC Tech. Pap., No 12, 29 pp.

FAHEY G. C. Jr., 1979. The nutritional significance of chemically defined dietary fibers, 117-146. In INGLETT G. E., FALKEHAG S. I., Dietory fibers, Chemistry and Nutrition, Acad. Press, New* York.

FENCHEL T. M., Mc ROY C. P., OGDEN J. C., PARKER P., RAINEY W. E., 1979. Symbiotic cellulose degradation in green turtles, Chelonia mydas L. Appl. environ. Microbiol, 37, 348-350.

FOLCH J., LEES M., SLOANE STANLEY G. H., 1957. A simple method for the isolation and purification of total lipids from animal tissues. J. biol. Chem., 226, 497-509.

GIGER S., SAUVANT D., DORLEANS M., MORAND-FEHR P., $1979 . \quad$ Détermination semi-automatique des constituants membranaires des aliments concentrés par la méthode de Van Soest. 30th annu. Meet. Eur. Assoc. Anim. Prod., Harrogate, England, 23-26 july 1979.

HALLIWELL G., 1959. The enzymic decomposition of cellulose. Nutr. Abstr. Rev., 29, 747-759.

HENRY Y., ETIENNE M., 1969. Effets nutritionnels de l'incorporation de cellulose purifiée dans le régime du porc en croissance-finition. Ann. Zootech., 18, 337-357.

HORSLEY R. W., 1977. A review of the bacterial flora of teleosts and elasmobranchs, including methods for its analysis. J. Fish Biol., 10, 529-553.

JOUANY J. P., 1978. Contribution à l'étude des protozoaires ciliés du rumen : leur dynamique, leur rôle dans la digestion et leur intérêt pour le ruminant. Th. Doct. Etat, Univ. Clermont II, 2 vol., 345 pp.

LANG J. A., BRIGGS G. M., 1976. The use and function of fiber in diets of monogastric animals, 151-169. In SPILLER G. A., AMEN R. J., Fiber in human nutrition, Plenum Press, New-York.

LESEL R., 1979. Données sur la microflore du tube digestif de la truite arc-en-ciel. Congr. nat. Ass. fr. Limnol., Marseille, 28-31 mai, p. 24, (résumé).

LUQUET P., 1971. Efficacité des protéines en relation avec leur faux d'incorporation dans l'alimentation de la truite arc-en-ciel. Ann. Hydrobiol., 2, 175-186.

NIEDERHOLZER R., HOFER R., 1979. The adaptation of digestive enzymes to temperature, season and diet in roach Rutilus rutilus L. and rudd Scardinius erythrophtalmus L. Cellulase. J. Fish Biol., 15, 411-416.

PARIS H., MURAT J. C., CASTILLA C., 1977. Etude des acides gras volatils dans l'intestin de trois espèces de poissons Téléostéens. C. R. Soc. Biol., 171, 1297-1301.

PREJS A., BLASZCZYK M., 1977. Relationships between food and cellulase activity in freshwater fishes. J. Fish Biol., 11, 447-452.

ROBERTSON J.B., VAN SOEST P. J., 1977. Dietary fiber estimation in concentrate feedstuffs. Am. Soc. anim. Sci. Madison (Wisconsin), July 23-27, 69, Doc 636, 2 pp.

SA WARDEKER J. S., SLONEKER J. H., JEANES A., 1965. Quantitative defermination of monosaccharides as their alditol acetates by gas liquid chromatography. Anal. Chem., 37, 1602-1604.

SMITH R. R., 1971. A method for measuring digestibility and metabolizable energy of fish feeds. Progve Fish Cult., 33, 132-134.

STICKNEY R. R., SHUMWAY S. E., 1974. Occurence of cellulase activity in the stomach of fishes J. Fish Biol., 6, 779-790. 
TANDLER A., BEAMISH W. H., 1979. Mechanical and biochemical components of apparent specific dynamic action in largemouth bass, Micropterus salmoides Lacépède. J. Fish Biol., 14, 343. 350.

TRUST T. J., BULL L. M., CURRIE B. R., BUCKLEY J. T., 1979. Obligate anaerobic bacteria in the gastrointestinal microflora of the grass carp (Ctenopharyngodon idella), goldfish (Carassius ouratus), and rainbow trout (Salmo gairdneri). J. Fish. Res. Board Can., 36, 1174-1179.

TRUST T. J., SPARROW R. A. H., 1974. The bacterial flora in the alimentary tract of freshwater salmonid fishes. Can. J. Microbiol., 20, 1219-1228.

VAN SOEST P. J., 1963. Use of detergents in the analysis of fibrous feeds. II. A rapid method for the determination of fiber and lignin. J. Assoc. off. agric. Chem., 46, 829-835.

VAN SOEST P. J., 1973. The uniformity and nutritive availibility of cellulose. Fed. Proc. Fed. am. Soc exp. Biol., 32, 1804-1808.

VAN SOEST P. J., WINE R. H., 1967. Use of detergents in the analysis of fibrous feeds. IV. Determination of plant cell-wall constituents. J. Assoc. off. agric. Chem., 50, 50-55. 